\title{
Ishak Score 4
}

National Cancer Institute

\section{Source}

National Cancer Institute. Ishak Score 4. NCI Thesaurus. Code C95154.

Marked bridging fibrosis (portal to portal and porto-central) is present. 